Article

\title{
A Population Based Study of Liver Function amongst Adults with Hyperuricemia and Gout in the United States
}

\author{
Subrata Deb ${ }^{1}$ iD and Prashant Sakharkar ${ }^{2, *}$ \\ 1 Department of Pharmaceutical Sciences, College of Pharmacy, Larkin University, Miami, FL 33169, USA; \\ sdeb@alumni.ubc.ca \\ 2 Clinical and Administrative Sciences, Roosevelt University College of Science, Health and Pharmacy, \\ Schaumburg, IL 60173, USA \\ * Correspondence: psakharkar@roosevelt.edu; Tel.: +1-847-240-4077
}

check for updates

Citation: Deb, S.; Sakharkar, P. A Population Based Study of Liver Function amongst Adults with Hyperuricemia and Gout in the United States. Diseases 2021, 9, 61. https://doi.org/10.3390/diseases9030061

Academic Editor:

Ludovico Abenavoli

Received: 14 July 2021

Accepted: 13 September 2021

Published: 17 September 2021

Publisher's Note: MDPI stays neutral with regard to jurisdictional claims in published maps and institutional affiliations.

Copyright: (c) 2021 by the authors. Licensee MDPI, Basel, Switzerland. This article is an open access article distributed under the terms and conditions of the Creative Commons Attribution (CC BY) license (https:// creativecommons.org/licenses/by/ $4.0 /)$

\begin{abstract}
To examine the association between uric acid levels and liver enzyme functions amongst adults with hyperuricemia and gout in the United States. The National Health and Nutrition Examination Survey (NHANES) data from 2007 to 2016 was used to study the research objective. Data were analyzed for descriptive statistics and for differences using the $t$ test, Chi-square test and ANOVA. A regression analysis was performed to determine association between demographics and liver enzymes. A $p$ value of $<0.05$ or $<0.001$ was considered statistically significant. A total of 14,946 adults ( $\geq 20$ yrs.) were included in this study. Sample mean age was $49 \pm 0.15$ yrs., and $54 \%$ were female. Overall, $15 \%$ adults had elevated uric acid levels ( $\geq 6.8 \mathrm{mg} / \mathrm{dL})$, men had significantly higher uric acid levels than women $(6 \mathrm{mg} / \mathrm{dL}$ vs. $4.8 \mathrm{mg} / \mathrm{dL})$. High uric acid levels were associated with more than two times higher odds of elevated ALT, AST and GGT $(p<0.001)$. Similarly, gender-based target uric acid values were associated with two-fold increased odds of GGT, over one-and-a-half fold higher odds of ALT and AST $(p<0.001)$. Regression analysis showed significant association between age, gender, race/ethnicity, body mass index, and hypertension and ALT, AST, ALP, total bilirubin and GGT $(p<0.001)$. Adults with hyperuricemia and gout are most likely to develop liver dysfunctions and suffer associated morbidities. Such patients need to be appropriately monitored and managed for their liver functions and to prevent associated morbidities.
\end{abstract}

Keywords: hyperuricemia; gout; NHANES; liver dysfunction; association

\section{Introduction}

The prevalence of hyperuricemia and gout in US population is approximately $20 \%$ and 4\%, respectively [1]. According to Chen-Xu et al. (2019), a staggering 47.1 million adults were estimated to be diagnosed with hyperuricemia considering gender-specific uric acid levels in 2015-2016 [1]. Though there is considerable variability in the definition of hyperuricemia, uric acid levels $>6.8 \mathrm{mg} / \mathrm{dL}$ is widely accepted as hyperuricemia in the general population [2]. However, there are gender-specific differences in the hyperuricemia criteria with serum urate levels of $\geq 6.8 \mathrm{mg} / \mathrm{dL}$ for men and $\geq 5.7 \mathrm{mg} / \mathrm{dL}$ for women designated as elevated levels [1]. Hyperuricemia or elevated levels of uric acid is a metabolic disorder that is known to be a major precursor for an inflammatory condition called gout. The formation of urate crystals from elevated uric acid levels and subsequent precipitation of those crystals trigger development of gout [3]. Although not all adults with hyperuricemia experience gout-related symptoms and progression. The inflammatory arthritis which is primarily caused by hyperuricemia is the primary symptoms of gout. Several comorbidities including overweight, hyperglycemia, elevated blood pressure and renal disorder contribute to hyperuricemia [3]. About $70 \%$ of the uric acid is renally excreted and thus certain drugs and increased concentrations of lead, lactates and ketones can block the renal excretion of uric acid, leading to accumulation of serum uric acid and development of hyperuricemia [4]. 
Liver function is critical for biosynthesis/metabolism of endogenous compounds as well as elimination of xenobiotics. Hepatic stress from endogenous or exogenous substances initiates liver cell growth which can stimulate liver enzymes such as alanine aminotransferases (ALT), aspartate aminotransferases (AST) and alkaline phosphatase (ALP) [5]. For uric acid biosynthesis, liver is the primary site with the highest protein expression of xanthine oxidase which is the main enzyme responsible for uric acid formation [6]. Thus, hepatocytes are consistently exposed to uric acid at a very high level. Cell culture studies with hepatocytes suggest that uric acid has the capability to cause mitochondrial oxidative stress and thus potentially trigger liver dysfunction [7]. Despite a high number of individuals experiencing hyperuricemia, there is limited information available about the effect of hyperuricemia on liver function in population-based observational studies. The goal of the present population-based cross-sectional study was to analyze liver function amongst adults with hyperuricemia and gout in the United States.

\section{Methods}

\subsection{Study Population}

We analyzed the sample of adults 20 years and older who participated in the National Health and Nutrition Examination Survey (NHANES), an ongoing population-based statistical survey designed to assess the health and nutritional status of adults and children in the US. NHANES uses a representative sample of the noninstitutionalized US civilian population that is selected using a multistage, stratified sampling design. The survey is unique in that it combines interviews, physical examinations and various laboratory data. In-person interviews were conducted in sampled households, and subjects were invited to participate in medical examinations. We extracted data on individuals who participated in NHANES from 2007 through 2016 into a combined dataset (NHANES 2007-2016) to increase sample size for greater estimator reliability (NHANES Analytic Guidelines) [8]. Of the total 51,694 participants on whom information was gathered during interview, $48,710(94 \%)$ were also screened for laboratory data. We excluded individuals $(26,983)$ who either had hepatitis B surface antibody $(n=12,599)$, hepatitis B surface antigen (159), hepatitis D antibody (43) and hepatitis C confirmed antibody combined $(n=336)$, and hepatitis A antibody $(21,737)$ and hepatitis B core antibody $(n=2071)$. We also excluded participants who self-reported of having liver condition on the interview (1127) and with either missing AST, ALT, ALP, GGT, total bilirubin and uric acid levels $(n=19,797)$ leaving final adjusted sample of 14,946 participants of age 20 years old or above for the analyses.

\subsection{Covariates}

Covariates included age, gender, ethnicity/race, education, income, poverty level, Body Mass Index (BMI), Systolic Blood Pressure (SBP), Diastolic Blood Pressure (DBP). Age, ethnicity/race, income, knowledge of having gout was self-reported by the participants.

\subsection{Assessment of Gout and Hyperuricemia}

Timed endpoint method was used to measure the concentration of uric acid in serum, plasma, or urine. Uric acid is oxidized by uricase to produce allatoin and hydrogen peroxide. The hydrogen peroxide reacts with 4-aminoantipyrine (4-AAP) and 3,5-dichloro2-hydroxybenzene sulfonate (DCHBS) in a reaction catalyzed by peroxidase to produce a colored product. The system monitors the change in absorbance at $520 \mathrm{~nm}$ at a fixed time interval. The change in absorbance is directly proportional to the concentration of uric acid in the sample. Details of quality-control procedures have been published elsewhere (http:/ / www.cdc.gov/nchs/data/nhanes/nhanes3/cdrom/nchs/manuals/labman.pdf, accessed on 10 May 2021).

Our primary definition of gout and hyperuricemia was in accordance with the American College of Rheumatology 2020 Guidelines for the uric acid range [2]. Individuals were considered of having hyperuricemia if they either had uric acid level of $6.8 \mathrm{mg} / \mathrm{dL}$, which is the standard target level, or having gout if they answered yes to the question "Doctor 
ever told you that you had gout" in the home interview. We also examined the potential impact of alternative definitions of hyperuricemia that included gender-based distinction of serum uric acid level $\geq 6.8 \mathrm{mg} / \mathrm{dL}$ in male and $\geq 5.7 \mathrm{mg} / \mathrm{dL}$ in female. Uric acid values reported in $\mathrm{mg} / \mathrm{dL}$ can be converted to $\mu$ moles/liter by multiplying by 59.48 . Body mass index, a measure of obesity defined as weight in kilograms divided by height in meters squared, was categorized according to clinical guidelines set by the National Institute of Health [9] and hypertension was defined as blood pressure of 130/85 mmHg according to the National Cholesterol Education Program-Adult Treatment Panel-III (NCEP-ATP III) guidelines [10].

\subsection{Statistical Analysis}

The statistical analyses for this study were performed using STATA ver14 (STATA Corp, College Station, TX, USA) a statistical software package that considers sample weighting and the complex, multistage probability sample design of NHANES [11]. Demographic characteristics were compared by age, gender, race/ethnicity, education and uric acid levels using the Chi-square test followed by post-hoc analyses with Bonferroni correction. Sampling weights were applied to account for selection probabilities, oversampling, nonresponse, and differences between the sample and the US adolescent male population. We examined the association between uric acid and liver enzyme levels. Logistic regression analyses were performed to quantify the magnitude of associations between the universal uric acid levels and gender-based uric acid levels and liver enzyme levels in presence of covariates. There is no universal agreement about the cut-off values for ALT and AST in published literature. In this study, we defined abnormal ALT and AST values as a range (<20 mg/dL, 20-29 mg/dL, 30-39 mg/dL and $\geq 40 \mathrm{mg} / \mathrm{dL}$ ) for total sample population. A threshold value of $17 \mathrm{mmol} / \mathrm{dL}$ for total bilirubin, $40 \mathrm{U} / \mathrm{L}$ for GGT and AST/ALT ratio of 1 was used. We also used a gender-based target value of ALT ( $\geq 30 \mathrm{U} / \mathrm{L}$ for male and $\geq 25 \mathrm{U} / \mathrm{L}$ for female) and AST ( $\geq 33 \mathrm{U} / \mathrm{L}$ for male and female both), and GGT ( $\geq 65 \mathrm{IU} / \mathrm{L}$ for male, $\geq 36 \mathrm{IU} / \mathrm{L}$ for female) to explore if this change in target value produces any difference in associations [12]. These target values were chosen as they represent common institutional reference values and generally used in the clinical practice. Similar target values have been used in both adolescent and adult in earlier epidemiological studies [13,14]. Regression model was adjusted for age, gender, ethnicity, BMI, hypertension and uric acid level. Taylor series linearization was used for variance estimation. A $p$ value of $\leq 0.05$ or $\leq 0.001$ was considered statistically and highly statistically significant, respectively.

\section{Results}

\subsection{Study Participants}

Table 1 presents the demographic characteristics of individuals with gout and hyperuricemia. A total of 14,946 adults (20 years and above) were included in this study. The mean age of the sample population was $49 \pm 0.15$ yrs. and $52 \%$ individuals were between age 20 and 40 years, 54\% were female, 55\% were non-Hispanic White, and $60 \%$ had some college or graduate level education. A total of $60 \%$ individuals had family income of less than USD 55,000,67\% were overweight and obese, 27\% had hypertension and $84 \%$ were taking prescription medication for their hypertension (Table 1). The mean BMI was $31 \mathrm{~kg} / \mathrm{m}^{2}$ and waist circumference was $106.1 \mathrm{~cm}$ in individuals with hyperuricemia, above the target value of 30 for BMI and waist circumference of $102 \mathrm{~cm}$ for men, and $88 \mathrm{~cm}$ for women (Table 1). Fifteen percent of adults had uric acid level $6.8 \mathrm{mg} / \mathrm{dL}$ in overall sample. Men had significantly higher serum uric acid levels than women $(6 \mathrm{mg} / \mathrm{dL}$ vs. $4.8 \mathrm{mg} / \mathrm{dL})$. Mean serum uric acid level in overall sample among adults was $5.4 \mathrm{mg} / \mathrm{dL}$, whereas it was $7.8 \mathrm{mg} / \mathrm{dL}$ in hypouricemic individuals, quite higher than the threshold value of $6.8 \mathrm{mg} / \mathrm{dL}$ recommended by the American Rheumatology Association (Table 1). 
Table 1. Demographic Characteristics and Laboratory Data of NHANES participants (2007-2016).

\begin{tabular}{|c|c|c|c|c|}
\hline & \multicolumn{4}{|c|}{ Uric Acid } \\
\hline & $\begin{array}{c}\text { Total Sample } \\
\mathrm{N}=14,946\end{array}$ & $\begin{array}{c}\text { Normal } \\
n=12,714(85.0)\end{array}$ & $\begin{array}{c}\text { Elevated } \\
n=2232(15.0)\end{array}$ & $p$-Value \\
\hline Age (yrs.) (Mean \pm SE) & $49.3(0.15)$ & $48.2(0.16)$ & $51.4(0.40)$ & $<0.001^{* *}$ \\
\hline \multicolumn{5}{|c|}{ Age (yrs.) } \\
\hline $20-44$ & $6447(51.9)$ & $5712(52.9)$ & $735(45.5)$ & $<0.001^{* *}$ \\
\hline $45-64$ & $4771(29.2)$ & $4080(29.3)$ & $691(28.3)$ & \\
\hline$\geq 65$ & 3728 (18.9) & $2922(17.8)$ & $806(26.2)$ & \\
\hline \multicolumn{5}{|c|}{ Gender } \\
\hline Male & $7037(45.8)$ & $5539(44.1)$ & $1678(76.2)$ & $<0.001^{* *}$ \\
\hline Female & 7909 (54.2) & 7355 (58.9) & $554(23.8)$ & \\
\hline \multicolumn{5}{|c|}{ Marital Status } \\
\hline Single & $2820(21.5)$ & 2447 (21.5) & $373(21.2)$ & 0.960 \\
\hline Married & $7664(52.6)$ & $6490(52.5)$ & $1174(52.9)$ & \\
\hline Other & $4456(25.9)$ & $3771(26.0)$ & 685 (25.9) & \\
\hline \multicolumn{5}{|c|}{ Race/Ethnicity } \\
\hline Mexican American & $3300(14.2)$ & $2916(14.5)$ & $384(12.2)$ & $<0.001^{* *}$ \\
\hline Other Hispanic & $2105(8.7)$ & $1856(8.8)$ & $249(7.3)$ & \\
\hline Non-Hispanic White & $4324(55.3)$ & $3637(53.2)$ & $687(54.4)$ & \\
\hline Non-Hispanic black & $3020(12.3)$ & $2428(11.9)$ & $592(15.1)$ & \\
\hline Other & 2197 (11.5) & 1877 (11.6) & $320(11.0)$ & \\
\hline \multicolumn{5}{|c|}{ Education } \\
\hline$<9$ th Grade & $2087(9.6)$ & 2087 (9.6) & $384(11.2)$ & $<0.001^{* *}$ \\
\hline 9-11th Grade & $1935(12.0)$ & $1935(12.0)$ & $364(12.8)$ & \\
\hline High School/GED & $2565(19.0)$ & $2565(19.0)$ & $515(24.3)$ & \\
\hline $\begin{array}{c}\text { Some College or } \\
\text { AA degree }\end{array}$ & 3305 (29.9) & 3305 (29.9) & $556(28.8)$ & \\
\hline $\begin{array}{c}\text { College Graduate } \\
\text { or above }\end{array}$ & $2808(29.5)$ & $2808(29.5)$ & $409(22.9)$ & \\
\hline \multicolumn{5}{|c|}{ Family Income } \\
\hline$<$ USD 25,000 & $5078(28.4)$ & $4270(28.2)$ & $808(29.9)$ & $0.020 *$ \\
\hline USD 25,000-USD 54,999 & $4690(31.6)$ & $3997(31.4)$ & $693(32.8)$ & \\
\hline USD 55,000-USD 99,999 & $2445(20.1)$ & 2074 (19.9) & $371(20.9)$ & \\
\hline >USD 100,000 & $1882(19.9)$ & $1652(20.4)$ & $230(16.4)$ & \\
\hline \multicolumn{5}{|c|}{ Ratio of Family Income to Poverty } \\
\hline$<1.35$ & $5108(28.6)$ & 4357 (28.6) & $751(28.4)$ & $0.027^{*}$ \\
\hline $1.35-1.84$ & $1717(10.8)$ & $1440(10.7)$ & $277(11.6)$ & \\
\hline $1.85-2.99$ & $2401(17.9)$ & $2021(17.5)$ & $380(20.3)$ & \\
\hline$\geq 3.00$ & 4219 (42.7) & $3626(43.2)$ & $593(39.7)$ & \\
\hline
\end{tabular}


Table 1. Cont.

\begin{tabular}{|c|c|c|c|c|}
\hline & \multicolumn{4}{|c|}{ Uric Acid } \\
\hline & $\begin{array}{c}\text { Total Sample } \\
\mathrm{N}=14,946\end{array}$ & $\begin{array}{c}\text { Normal } \\
n=12,714(85.0)\end{array}$ & $\begin{array}{c}\text { Elevated } \\
n=2232(15.0)\end{array}$ & $p$-Value \\
\hline \multicolumn{5}{|c|}{ BMI $\left(\mathrm{kg} / \mathrm{m}^{2}\right)$} \\
\hline Underweight & $240(1.8)$ & $229(2.0)$ & $11(0.5)$ & $<0.001 * *$ \\
\hline Normal & $4301(31.5)$ & $3972(34.2)$ & $329(14.3)$ & \\
\hline Overweight & $5021(33.1)$ & $4306(33.2)$ & $715(32.6)$ & \\
\hline Obese & $5210(33.6)$ & $4073(30.6)$ & $1137(52.6)$ & \\
\hline \multicolumn{5}{|c|}{ SBP } \\
\hline$<135 \mathrm{mmHg}$ & $9903(75.1)$ & $8642(76.7)$ & $1261(65.2)$ & $<0.001$ ** \\
\hline$\geq 135 \mathrm{mmHg}$ & $4474(24.9)$ & $3577(25.3)$ & $897(34.8)$ & \\
\hline \multicolumn{5}{|c|}{ DBP } \\
\hline$<85 \mathrm{mmHg}$ & $13,186(92.7)$ & $11,303(93.6)$ & $1883(87.1)$ & $<0.001 * *$ \\
\hline$\geq 85 \mathrm{mmHg}$ & $1191(7.3)$ & $916(6.4)$ & $275(12.9)$ & \\
\hline \multicolumn{5}{|c|}{ Hypertension (135/85 mmHg) } \\
\hline Yes & $9633(73.2)$ & $8834(75.0)$ & $1199(61.9)$ & $<0.001 * *$ \\
\hline No & $4744(26.8)$ & $3785(25.0)$ & $959(38.1)$ & \\
\hline \multicolumn{5}{|c|}{ Taking prescription for hypertension } \\
\hline Yes & $4447(84.1)$ & $3388(83.6)$ & $1059(85.9)$ & 0.208 \\
\hline No & $622(15.9)$ & $511(16.4)$ & $111(14.1)$ & \\
\hline $\begin{array}{l}\mathrm{BMI}\left(\mathrm{kg} / \mathrm{m}^{2}\right) \\
(\text { Mean } \pm \mathrm{SE})\end{array}$ & $28.6(0.05)$ & $28.1(0.06)$ & $31.1(0.14)$ & $<0.001 * *$ \\
\hline $\begin{array}{l}\text { Waist Circumference } \\
(\mathrm{cm})(\text { Mean } \pm \text { SE })\end{array}$ & $97.5(0.13)$ & $96(0.14)$ & $106.1(0.34)$ & $<0.001^{* *}$ \\
\hline $\begin{array}{l}\mathrm{SBP}(\mathrm{mmHg}) \\
(\mathrm{Mean} \pm \mathrm{SE})\end{array}$ & $123.8(0.2)$ & $122.9(0.17)$ & $129.0(0.42)$ & $<0.001 * *$ \\
\hline $\begin{array}{l}\mathrm{DBP}(\mathrm{mmHg}) \\
(\text { Mean } \pm \mathrm{SE})\end{array}$ & $69.1(0.11)$ & $68.9(0.11)$ & $70.1(0.32)$ & $<0.001$ ** \\
\hline $\begin{array}{l}\text { Uric Acid }(\mathrm{mg} / \mathrm{dL}) \\
\quad(\text { Mean } \pm \mathrm{SE})\end{array}$ & $5.4(0.01)$ & $5.0(0.01)$ & $7.8(0.02)$ & $<0.001^{* *}$ \\
\hline
\end{tabular}

Data presented as frequency and percent in parentheses unless specified; Normal uric acid level $(<6.8 \mathrm{mg} / \mathrm{dL})$ Elevated uric acid level ( $\geq 6.8 \mathrm{mg} / \mathrm{dL})$; BMI: Body Mass Index; SBP: Systolic blood pressure; DBP: Diastolic blood pressure; Significant at ${ }^{*} p<0.05$ and ${ }^{* *} p<0.001$.

\subsection{Association of Participants Characteristics and Uric Acid Levels}

We examined the association of demographic characteristics with serum uric acid level using the Chi-square test. High serum uric acid levels were significantly associated with age, gender, race/ethnicity, education and family income. Individuals of age 45 years and above and being male showed significant association with higher serum uric acid levels. In general, among both men and women, the higher uric acid levels were associated with an increasing blood pressure and body mass index. Individuals with history of hypertension had a significantly higher serum uric acid level than those who did not. Among individuals with gout and hyperuricemia, 76\% were male, 54\% were non-Hispanic White, $61 \%$ had hypertension and $85 \%$ were on prescription medication for hypertension and 53\% were obese. These proportions were substantially and significantly higher than those among individuals without gout and hyperuricemia (Table 1). 


\subsection{Association of Uric Acid Levels with Abnormal Liver Enzymes}

We also examined the association of serum uric acid levels with liver enzymes. Uric acid levels were found to be significantly associated with liver enzymes ALT and AST, AST / ALT ratio and total bilirubin (Table 2). Post hoc analysis showed high uric acid level was significantly associated with high ALT $(\geq 30)$, AST $(\geq 33)$ and total bilirubin levels $(\geq 17)$ except for ALP. Forty percent of individuals had high ALT $(\geq 30), 31 \%$ had high AST ( $\geq 33$ ) and $23 \%$ had high total bilirubin levels ( $\geq 17$ ) (Table 2). High uric acid levels were also found significantly associated with gender-based target values for ALT $\geq 30 \mathrm{U} / \mathrm{L}$ in male and $\geq 25 \mathrm{U} / \mathrm{L}$ in female, $33 \mathrm{U} / \mathrm{L}$ in both male and female for AST and $\geq 65 \mathrm{U} / \mathrm{L}$ in male and $\geq 36 \mathrm{U} / \mathrm{L}$ in female for GGT compared to normal uric acid levels. Thirteen percent of individuals had high ALT, $22 \%$ had high AST and $42 \%$ had high total bilirubin levels (Table 3).

Table 2. Association between Hyperuricemia and Liver Enzymes.

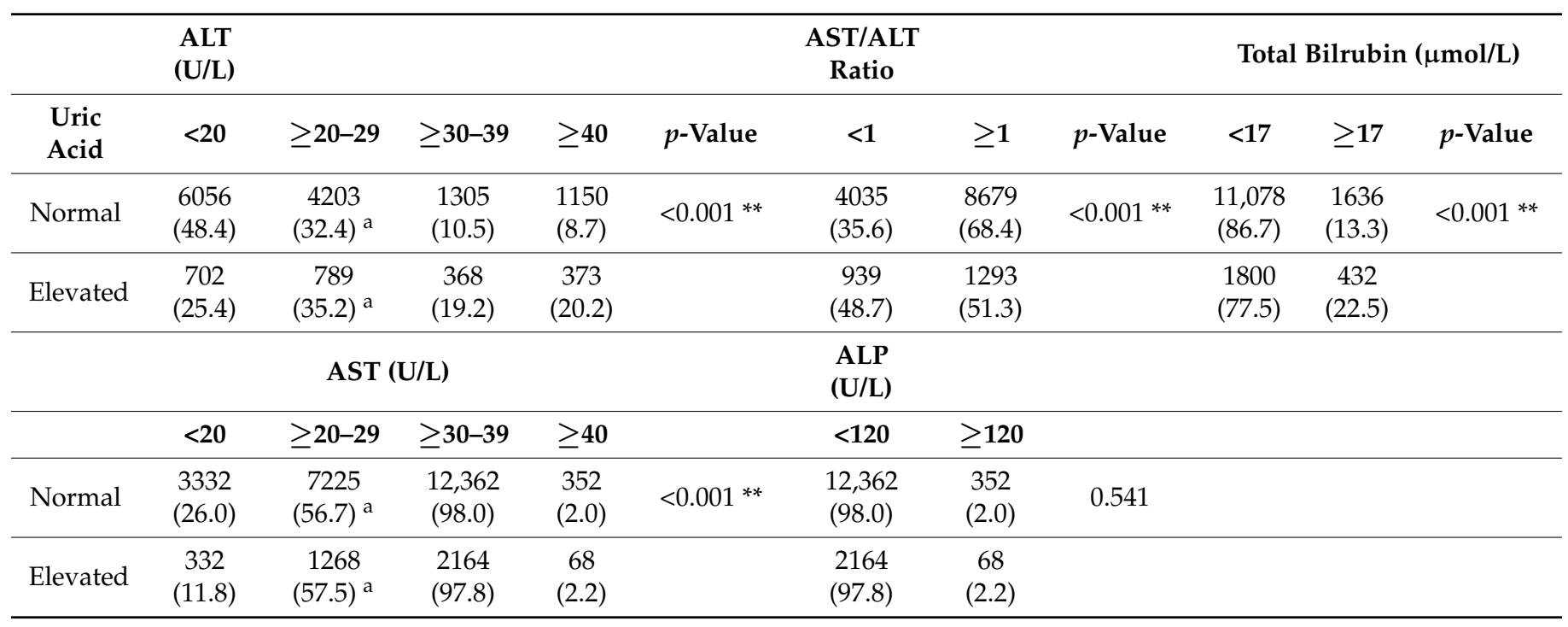

Data presented as frequency and percent in parentheses. Normal uric acid level $(<6.8 \mathrm{mg} / \mathrm{dL})$; Elevated uric acid level $(\geq 6.8 \mathrm{mg} / \mathrm{dL}) ;$ Significant at ${ }^{* *} p<0.001$. ${ }^{a}$ Subscript letter denotes category whose column proportions did not differ significantly from others on post hoc analyses with Bonferroni correction.

Table 3. Association between Hyperuricemia and Liver Enzymes (Gender-based cut-off value).

\begin{tabular}{|c|c|c|c|}
\hline \multicolumn{4}{|c|}{ ALT (U/L) } \\
\hline Uric Acid (mg/dL) & Normal & Elevated & $p$-Value \\
\hline Normal & $11,562(92.5)$ & $1152(7.5)$ & $<0.001^{* *}$ \\
\hline Elevated & $1942(87.2)$ & $290(12.8)$ & \\
\hline Uric Acid (mg/dL) & \multicolumn{2}{|c|}{ AST (U/L) } & \\
\hline & Normal & Elevated & \\
\hline Normal & $11,276(89.4)$ & 1438 (10.6) & $<0.001^{* *}$ \\
\hline Elevated & $1785(78.1)$ & 447 (21.9) & \\
\hline \multirow[t]{2}{*}{ Uric Acid (mg/dL) } & \multicolumn{2}{|c|}{ GGT (U/L) } & \\
\hline & Normal & Elevated & \\
\hline Normal & $9579(75.8)$ & $3135(24.2)$ & $<0.001 * *$ \\
\hline Elevated & 1437 (58.5) & 795 (41.5) & \\
\hline
\end{tabular}

Data presented as frequency and percent in parentheses; Normal uric acid level $(<6.8 \mathrm{mg} / \mathrm{dL})$; Elevated uric acid level ( $\geq 6.8 \mathrm{mg} / \mathrm{dL}$ ); Normal ALT ( $<30 \mathrm{U} / \mathrm{L}$ for Male and $<25 \mathrm{U} / \mathrm{L}$ for Female); Elevated ALT ( $\geq 30 \mathrm{U} / \mathrm{L}$ for Male and $\geq 25 \mathrm{U} / \mathrm{L}$ for Female); Normal AST ( $<33 \mathrm{U} / \mathrm{L}$ for both Male and Female); Elevated AST ( $\geq 33 \mathrm{U} / \mathrm{L}$ for both Male and Female); Normal GGT ( $<65 \mathrm{U} / \mathrm{L}$ for Male and $<36 \mathrm{U} / \mathrm{L}$ for Female); Elevated GGT ( $\geq 65 \mathrm{U} / \mathrm{L}$ for Male and $\geq 36 \mathrm{U} / \mathrm{L}$ for Female); Significant at ${ }^{* *} p<0.001$. 
We also calculated the odds ratio of abnormal ALT, AST, AST/ALT ratio, ALP, GGT and total bilirubin using both overall and gender-based target value of serum uric acid. Uric acid levels were associated with over two times higher odds of elevated ALT (OR: 2.22, 95\%CI: 1.94, 2.54, $p<0.001$ ), AST (OR: 2.36, 95\% CI: 2.02, 2.77, $p<0.001$ ), GGT (OR: $2.20,95 \%$ CI: $1.88,2.57, p<0.001)$ and over one-and-a-half times of higher odds of total bilirubin (OR: 1.89, 95\% CI: 1.64, 2.18, $p<0.001$ ) (Table 4). Gender-based target values of uric acid were associated with two-fold increased odds of GGT (OR: 2.26, 95\%CI: 1.90, 2.69, $p<0.001$ ), over one and half fold higher odds of ALT (OR: 1.90, 95\%CI: 1.70, 2.11, $p<0.001$ ), AST (OR:1.84, 95\%CI: 1.59, 2.13, $p<0.001$ ) whereas, over one times higher odds of total bilirubin (OR:1.29, 95\%CI: 1.13, 1.47, $p<0.001$ ) (Table 4).

Table 4. Odds of Elevated Liver Enzymes with Uric acid (Universal and gender-based cut-off value).

\begin{tabular}{|c|c|c|c|c|c|c|}
\hline \multirow{2}{*}{$\begin{array}{l}\text { Uric Acid } \\
\text { (mg/dL) }\end{array}$} & \multicolumn{2}{|c|}{ ALT (U/L) } & \multicolumn{2}{|c|}{ AST (U/L) } & \multicolumn{2}{|c|}{ AST/ALT Ratio } \\
\hline & OR $(95 \% C I)$ & $p$-Value & OR $(95 \% C I)$ & $p$-Value & OR $(95 \% \mathrm{CI})$ & $p$-Value \\
\hline Universal & $2.22(1.94,2.54)$ & $<0.001 * *$ & $2.36(2.02,2.77)$ & $<0.001^{* *}$ & $0.49(0.42,0.56)$ & $<0.001^{* *}$ \\
\hline \multirow[t]{2}{*}{ Gender-based } & $1.90(1.70,2.11)$ & $<0.001^{* *}$ & $1.84(1.59,2.13)$ & $<0.001^{* *}$ & $0.64(0.58,0.72)$ & $<0.001^{* *}$ \\
\hline & \multicolumn{2}{|c|}{$\operatorname{ALP}(\mathrm{U} / \mathrm{L})$} & \multicolumn{2}{|c|}{ GGT (U/L) } & \multicolumn{2}{|c|}{ Total Bilirubin $(\mu \mathrm{mol} / \mathrm{L})$} \\
\hline $\begin{array}{l}\text { Uric Acid } \\
\text { (mg/dL) }\end{array}$ & OR $(95 \% C I)$ & $p$-Value & OR $(95 \% C I)$ & $p$-Value & OR $(95 \% C I)$ & $p$-Value \\
\hline Universal & $1.11(0.80,1.53)$ & 0.542 & $2.20(1.88,2.57)$ & $<0.001 * *$ & $1.89(1.64,2.18)$ & $<0.001^{* *}$ \\
\hline Gender-based & $1.09(0.82,1.47)$ & 0.539 & $2.26(1.90,2.69)$ & $<0.001^{* *}$ & $1.29(1.13,1.47)$ & $<0.001^{* *}$ \\
\hline
\end{tabular}

Elevated universal $(\geq 6.8 \mathrm{mg} / \mathrm{dL})$; Elevated gender-based ( $\geq 6.8 \mathrm{mg} / \mathrm{dL}$ in male and $\geq 5.7 \mathrm{mg} / \mathrm{dL}$ in female); OR = Odds Ratio; $\mathrm{CI}=\mathrm{Confi}-$ dence Interval; Significant at ${ }^{* *} p<0.001$.

\subsection{Association of Gender-Based Target Values of Uric Acid with Abnormal Liver Enzymes}

Gender based target values of serum uric acid levels were also found significantly associated with ALT, AST, AST/ALT ratio and total bilirubin. Gender-based target values of serum uric acid were also found to be associated and gender-based target values of ALT ( $\geq 30 \mathrm{U} / \mathrm{L}$ in male and $\geq 25 \mathrm{U} / \mathrm{L}$ in female), AST (33 U/L in both male and female) and GGT ( $\geq 65 \mathrm{U} / \mathrm{L}$ in male and $\geq 36 \mathrm{U} / \mathrm{L}$ in female).

\subsection{Predictors of High Liver Enzymes Levels on Regression Analyses}

We modeled the odds of abnormal ALT ( $\geq 30 \mathrm{U} / \mathrm{L}$ for male and $\geq 25 \mathrm{U} / \mathrm{L}$ for female) and AST ( $\geq 33 \mathrm{U} / \mathrm{L}$ for male and female both), AST / ALT ratio ( $\geq 1)$, ALP ( $\geq 120 \mathrm{U} / \mathrm{L})$, GGT ( $\geq 65 \mathrm{IU} / \mathrm{L}$ for male, $\geq 36 \mathrm{IU} / \mathrm{L}$ for female) and total bilirubin $(\geq 17 \mathrm{mmol} / \mathrm{dl}$ ) using uric acid and other covariates simultaneously and calculated the odds ratio using a logistic regression model (Table 5). On regression analysis, after adjustment, serum uric acid level was associated with higher ALT (OR:1.57, 95\%CI: 1.36, 1.80, $p<0.001)$, AST (OR:1.68, 95\%CI: $1.42,2.00, p<0.001)$, GGT (OR:1.86, 95\%CI: 1.47, 2.34, $p<0.001)$ and total bilirubin (OR:1.79, 95\%CI: 1.51, 2.12, $p<0.001)$. BMI was associated with higher ALT (OR:1.06, 95\%CI: 1.05, 1.07, $p<0.001$ ), AST (OR:1.02, 95\%CI: 1.01, 1.03, $p<0.001)$, ALP (OR:1.04, 95\%CI: 1.02, 1.05, $p<0.001$ ) and GGT levels (OR:1.04, 95\%CI: 1.03, 1.05, $p<0.001$ ). Hypertension was also associated with higher ALT (OR:1.19, 95\%CI: 1.03, 1.36, $p=0.015)$, AST (OR:1.27, 95\%CI: $1.08,1.50, p=0.005)$, ALP (OR:1.38, 95\%CI: 1.04, 1.84, $p=0.028)$ and GGT (OR:1.43, 95\%CI: $1.18,1.72, p<0.001)$. Age was also associated with high ALP (OR:1.02, 95\%CI: 1.00, 1.03, $p<0.001)$ and GGT (OR:1.01,95\%CI: 1.00, 1.01, $p<0.001)$. Female gender was associated with lower ALT (OR:0.50, 95\%CI: 0.98, 0.99, $p<0.001)$, AST (OR:0.45, 95\%CI: 0.40, 0.50, $p<0.001$ ) and total bilirubin (OR:0.37, 95\%CI: 0.37, 0.43, $p<0.001$ ), whereas with high GGT levels (OR:1.89, 95\%CI: 1.56, 2.29, $p<0.001$ ). Race/ethnicity was associated with low ALT (OR:0.86, 95\%CI: 0.81, 0.90, $p<0.001$ ), AST (OR:0.92, 95\%CI: 0.87, 0.96, $p<0.001$ ) and ALP 
(OR:0.80, 95\%CI: 0.72, 0.89, $p<0.001$ ). Other Hispanics had lower ALT, AST and ALP levels compared to another race/ethnicity (Table 5).

Table 5. Predictors of Liver Enzymes on Regression (Adjusted Model).

\begin{tabular}{|c|c|c|c|c|c|c|}
\hline & \multicolumn{2}{|c|}{ ALT (U/L) } & \multicolumn{2}{|c|}{ AST (U/L) } & \multicolumn{2}{|c|}{ AST/ALT Ratio } \\
\hline & OR $(95 \% \mathrm{CI})$ & $p$-Value & OR $(95 \% \mathrm{CI})$ & $p$-Value & OR $(95 \% \mathrm{CI})$ & $p$-Value \\
\hline Age & $0.99(0.98,0.99)$ & $<0.001 * *$ & $0.99(0.99,1.00)$ & 0.365 & $1.02(1.01,1.02)$ & $<0.001 * *$ \\
\hline Gender & $0.50(0.45,0.56)$ & $<0.001^{* *}$ & $0.45(0.40,0.50)$ & $<0.001^{* *}$ & $3.58(3.20,4.00)$ & $<0.001^{* *}$ \\
\hline Race & $0.86(0.81,0.90)$ & $<0.001^{* *}$ & $0.92(0.87,0.96)$ & $<0.001^{* *}$ & $1.18(1.13,1.23)$ & $<0.001^{* *}$ \\
\hline BMI & $1.06(1.05,1.07)$ & $<0.001^{* *}$ & $1.02(1.01,1.03)$ & $<0.001^{*}$ & $0.92(0.91,0.93)$ & $<0.001^{* *}$ \\
\hline HTN & $1.19(1.03,1.36)$ & $0.015 *$ & $1.27(1.08,1.50)$ & $0.005 *$ & $0.95(0.84,1.06)$ & 0.348 \\
\hline \multirow[t]{3}{*}{ Uric Acid } & $1.57(1.36,1.80)$ & $<0.001 * *$ & $1.68(1.42,2.00)$ & $<0.001^{* *}$ & $0.84(0.71,0.98)$ & $0.029 *$ \\
\hline & \multicolumn{2}{|c|}{ ALP (U/L) } & \multicolumn{2}{|c|}{ GGT (U/L) } & \multicolumn{2}{|c|}{ Total Bilirubin $(\mu \mathrm{mol} / \mathrm{L})$} \\
\hline & OR $(95 \% \mathrm{CI})$ & $p$-Value & OR $(95 \% \mathrm{CI})$ & $p$-Value & OR $(95 \% \mathrm{CI})$ & $p$-Value \\
\hline Age & $1.02(1.00,1.03)$ & $<0.001 * *$ & $1.01(1.00,1.01)$ & $<0.001^{* *}$ & $0.99(0.99,1.02)$ & 0.382 \\
\hline Gender & $1.34(0.96,1.88)$ & 0.082 & $1.89(1.56,2.29)$ & $<0.001 * *$ & $0.37(0.32,0.43)$ & $<0.001^{* *}$ \\
\hline Race & $0.80(0.72,0.89)$ & $<0.001^{* *}$ & $0.96(0.89,1.03)$ & 0.264 & $0.99(0.93,1.05)$ & 0.650 \\
\hline BMI & $1.04(1.02,1.05)$ & $<0.001^{* *}$ & $1.04(1.03,1.05)$ & $<0.001^{* *}$ & $0.93(0.92,0.94)$ & $<0.001^{* *}$ \\
\hline HTN & $1.38(1.04,1.84)$ & $0.028 *$ & $1.43(1.18,1.72)$ & $<0.001^{* *}$ & $0.96(0.81,1.13)$ & 0.608 \\
\hline Uric Acid & $1.00(0.66,1.53)$ & 0.967 & $1.86(1.47,2.34)$ & $<0.001 * *$ & $1.79(1.51,2.12)$ & $<0.001^{* *}$ \\
\hline
\end{tabular}

OR = Odds Ratio; CI = Confidence Interval; BMI: Body Mass Index; HTN: Hypertension (135/85 mmHg); Significant at * $p<0.05$ and ** $p<0.001$.

Similar pattern was observed when gender-based target serum uric acid level was used (Supplemental Figures S1 and S2). After adjustment, uric acid level was associated with higher ALT (OR:1.58, 95\%CI: 1.40, 1.77, $p<0.001$ ), AST (OR:1.60, 95\%CI: 1.37, 1.87, $p<0.001$ ), GGT (OR:1.81, 95\%CI: 1.52, 2.17, $p<0.001$ ) and total bilirubin (OR:1.63, 95\%CI: $1.42,1.88, p<0.001)$ Race/ethnicity was associated with low ALT (OR:0.82, 95\%CI: 0.81, $0.89, p<0.001$ ), AST (OR:0.91, 95\%CI: 0.87, 0.96, $p<0.001$ ) and ALP (OR:0.81, 95\%CI: 0.73, $0.90, p<0.001)$. Other Hispanics had lower ALT, AST and ALP levels compared to another race/ethnicity (Supplemental Table S1).

\section{Discussion}

Hyperuricemia is the primary cause of a condition called gout. In addition to inflammatory effects, uric acid can cause cellular toxicity [15]. In the present study, the participants from 2007 to 2016 NHANES study cycles were evaluated for availability of information on gout, uric acid level and liver enzyme function indicators. The main objective of this study was to examine any association among the uric acid levels and liver function as determined by AST, ALT and ALP in 14,946 adults with serum uric acid level and individuals with reported diagnosis of gout. Demographic characteristics indicate that the study population has a mean age of 49.3 with slightly over $50 \%$ of the study subjects were in the age range of 20 to 44 years with approximately equal representation of each sex in the sample. The results from our analyses suggest that adults $\geq 49$-years-old and being male had elevated serum uric acid levels. In general, majority of the participants had increased blood pressure, were overweight, and higher BMI. Similar to other metabolic disorders, hyperuricemia was also found to be associated with race/ethnicity, education, and family income.

Though there are gender-specific differences in the cut-off values for hyperuricemia, $\geq 6.8 \mathrm{mg} / \mathrm{dL}$ is considered a reasonable clinical target to avoid any gout-related complications [2]. In our NHANES samples, the mean uric acid levels were $5.4 \mathrm{mg} / \mathrm{dL}$ for the whole study population with mean level of $5.0 \mathrm{mg} / \mathrm{dL}$ among the group categorized as $<6.8 \mathrm{mg} / \mathrm{dL}$ and $7.8 \mathrm{mg} / \mathrm{dL}$ mean uric acid level in the group categorized as $\geq 6.8 \mathrm{mg} / \mathrm{dL}$. 
High liver function markers (AST $\geq 33$, ALT $\geq 30$, total bilirubin $\geq 17$ ) were significantly associated with high uric acid levels $(\geq 6.8 \mathrm{mg} / \mathrm{dL})$. The gender-based cut-off values for upper normal levels of AST, ALT and GGT was significantly associated with serum uric acid levels. The individuals with elevated uric acid levels had two times higher odds of abnormal AST, ALT, or GGT functions. Similarly, gender-based target values of uric acid indicated higher odds of getting diagnosed with elevated liver functions among the participants. Regression models suggest that uric acid is a good predictor of abnormal AST, ALT, GGT and total bilirubin levels. Similarly, age, gender, race and BMI covariates were also strongly associated with higher liver functions. Gender-based cut-off values of uric acid mirrored the results from the overall value of elevated uric acid ( $\geq 6.8 \mathrm{mg} / \mathrm{dL}$ ).

Previous population studies with subjects from earlier timelines or from different countries have highlighted the relationship between serum uric acid levels and a variety of liver dysfunction. Based on NHANES data from 1988-1994 and 1999-2006, Afzali and colleagues concluded that elevated uric acid levels pose a higher risk of developing liver cirrhosis and its related severity [16]. Likewise, analysis of 1988-1994 NHANES data in another study indicated that increased levels of uric acid was connected to non-alcoholic fatty liver disease (NAFLD) that was confirmed on ultrasound screening [17]. Interestingly, the cohort of subjects included in the study were nondiabetic, thus eliminating a major contributing factor to NAFLD [14] and highlighting the independent role of uric acid in NAFLD. In a cross-sectional study in China, elevated uric acid and obesity synergistically influenced the development and progression of NAFLD as determined by ultrasound screening [18]. Similarly, Shih et al., (2015) in their research reiterated the relationship between serum uric acid and NAFLD in US population [19]. In another study conducted among US pediatric patients, serum, uric acid levels were associated with nonalcoholic steatohepatitis potentially mirroring fructose intake by the children [20]. Catanzaro et al. (2020) reported an association between elevated uric acid and NAFLD in European Mediterranean individuals [21]. Incidentally, there have been quite a few cross-sectional and prospective observational studies with Chinese population analyzing serum uric acid and liver health. Bao et al. (2020) in their study identified a relationship between serum uric acid level and NAFLD in a population of non-obese post-menopausal women [22]. Similarly, in another prospective observational study, individuals with elevated serum uric acid levels were found to have a greater potential to develop NAFLD [23]. Several other population studies reported from Japan, Greece, Turkey, Korea and Israel indicated elevated serum uric acid as a potential risk factor for developing some form of liver dysfunction [24-28].

Liver dysfunction can result directly through elevated uric acid level through multidimensional mechanisms. It can induce inflammation and oxidative stress, leading to liver cell death and decrease in functional hepatocytes through lipids and glucose metabolism [7]. Interestingly, uric acid has the potential to cause oxidative stress and mitochondrial dysfunction directly [7]. Uric can also increase lipid synthesis by stimulating endoplasmic reticulum (ER) stress and though Sterol regulatory element-binding protein 1 (SREBP-1) and other transcriptional elements [29]. Increased lipid levels in turn can cause inflammation and oxidative stress. In addition, uric acid can promote cellular glucose biosynthesis which can catalyze increased inflammation in liver [6]. Thus, uric acid either directly or via lipids and glucose can induce inflammation and oxidative stress, leading to liver cell death and decrease in functional hepatocytes (Figure 1). These events reduce the liver's functional capacity resulting in elevated levels of liver enzyme functions (ALT, AST, ALP). As a secondary outcome of compromised liver function, the liver drug metabolizing capacity and ability to eliminate drugs can decrease. This can reflect in increased drug adverse effects and potential treatment failure [30]. It is worth recognizing that hyperuricemia is the driving force behind development of gout, a severe inflammatory condition [3]. Indeed, levels of several inflammatory markers including white blood cell, neutrophil count, Creactive protein, interleukin-6, interleukin-18 and tumor necrosis factor- $\alpha$ were higher in hypouricemic conditions [31]. The cellular and tissue alterations of liver are considered directly responsible for histopathogenesis of NAFLD including steatosis and non-alcoholic 
steatohepatitis [32,33]. In addition, recent reports suggest that hyperuricemia is a potential marker for cardio-metabolic disease including arrythmia [34,35]. Thus, the mechanism of uric acid-driven liver dysfunction can be complex and multifaceted and appears to occur through inflammation-related pathways. The results from the current study suggest that elevated serum uric acid levels are associated with abnormal liver function.

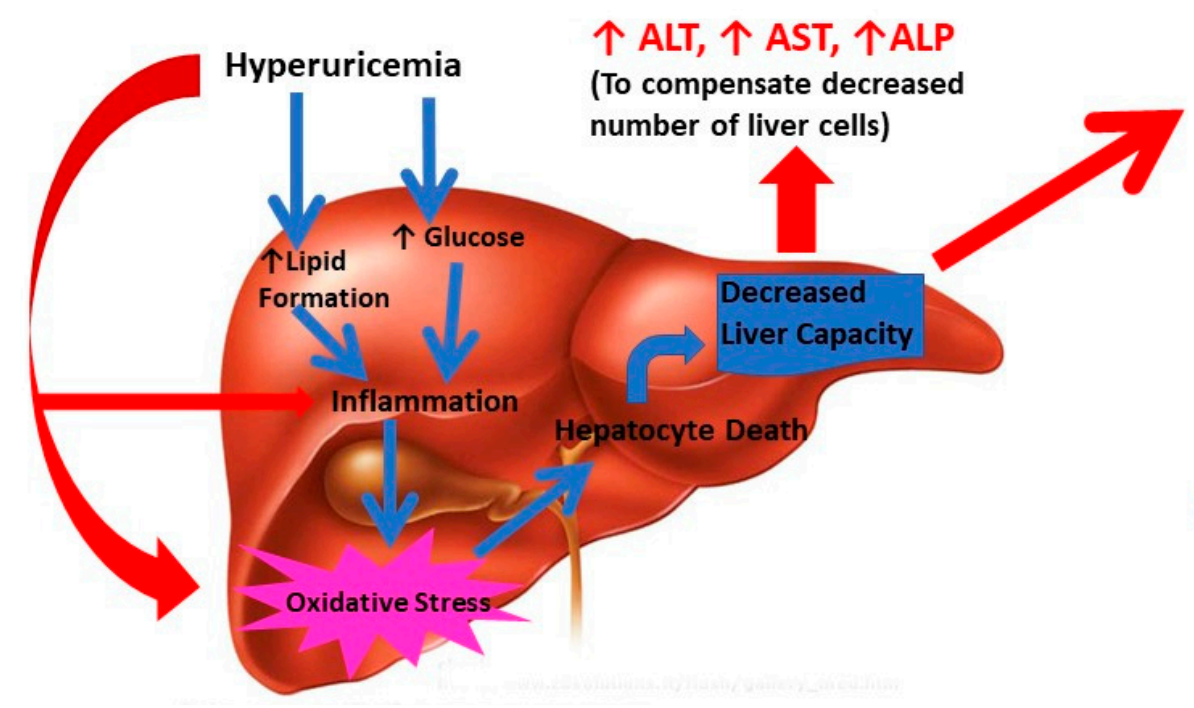

\section{Decreased Hepatic Drug Metabolizing Enzymes}

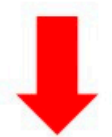

Reduced Elimination of Drugs

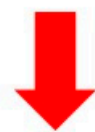

Increased Adverse Effects

Especially, from Narrow Therapeutic Index Drugs such as Warfarin, Phenytoin

Figure 1. Potential pathophysiological mechanism of hyperuricemia related liver dysfunction in gout.

Our study has several limitations even though it was performed using the current nationally representative sample of noninstitutionalized US civilian population. This study was not able to establish potential temporal relations between hyperuricemia and abnormal liver enzymes, which can be further addressed by longitudinal prospective studies. The diagnosis of gout by a physician among the participants was self-reported and could not be validated. However, it is unlikely that such misclassification would have provided such strong associations observed in this population study. We used liver enzyme levels only as a surrogate marker in the absence of liver ultrasound examination to assess the hepatic dysfunction. Furthermore, the serum uric acid levels in this study were ascertained objectively without reliance on participants' recall and showed consistent results.

\section{Conclusions}

Individuals with hyperuricemia and gout were at one to two times higher risk of having elevated liver enzyme levels. Men had significantly higher uric acid levels than women. Female had lower ALT, AST and total bilirubin, whereas they had higher GGT levels compared to men. Other Hispanics had lower ALT, AST and ALP levels compared to another race/ethnicity. Adults with hyperuricemia and gout need to be appropriately monitored and managed for their liver functions and to prevent associated morbidities.

Supplementary Materials: The following are available online at https:/ / www.mdpi.com/article/10 .3390 / diseases $9030061 /$ s1, Figure S1: Forest plots of the odds of elevated liver enzymes with hyperuricemia using universal cut-off value; Figure S2: Forest plots of the odds of elevated liver enzymes with hyperuricemia using gender-based cut-off values; Table S1: Predictors of Liver Enzymes on Regression (Adjusted Model).

Author Contributions: Conceptualization, P.S. and S.D.; methodology, P.S. and S.D.; software, P.S.; formal analysis, P.S.; data curation, P.S.; writing—original draft preparation, S.D. and P.S.; writingreview and editing, S.D. and P.S.; project administration, S.D. and P.S. All authors have read and agreed to the published version of the manuscript.

Funding: This research received no external funding. 
Institutional Review Board Statement: Ethical review and approval were neither sought nor applicable to this study since the NHANES data is available publicly for use. Use and analysis of deidentified publicly available data does not constitute human subjects research and as such does not require IRB review.

Informed Consent Statement: Not applicable.

Data Availability Statement: NHANES data is available in a publicly accessible repository through Centers for Disease Control and Prevention (CDC) and the National Center for Health Statistics (NCHS). The data presented in this study are openly available in the National Health and Nutrition Examination Survey (NHANES) website at https://wwwn.cdc.gov/nchs/nhanes/ (accessed on 14 July 2021).

Conflicts of Interest: The authors declare no conflict of interest.

\section{References}

1. Chen-Xu, M.; Yokose, C.; Rai, S.K.; Pillinger, M.H.; Choi, H.K. Contemporary Prevalence of Gout and Hyperuricemia in the United States and Decadal Trends: The National Health and Nutrition Examination Survey, 2007-2016. Arthritis Rheumatol. 2019, 71, 991-999. [CrossRef] [PubMed]

2. FitzGerald, J.D.; Dalbeth, N.; Mikuls, T.; Brignardello-Petersen, R.; Guyatt, G.; Abeles, A.M.; Gelber, A.C.; Harrold, L.R.; Khanna, D.; King, C.; et al. 2020 American College of Rheumatology Guideline for the Management of Gout. Arthritis Care Res. 2020, 72, 744-760. [CrossRef] [PubMed]

3. Dehlin, M.; Jacobsson, L.; Roddy, E. Global epidemiology of gout: Prevalence, incidence, treatment patterns and risk factors. Nat. Rev. Rheumatol. 2020, 16, 380-390. [CrossRef] [PubMed]

4. Chen, C.; Lu, J.M.; Yao, Q. Hyperuricemia-Related Diseases and Xanthine Oxidoreductase (XOR) Inhibitors: An Overview. Med. Sci. Monit. 2016, 22, 2501-2512. [CrossRef] [PubMed]

5. Contreras-Zentella, M.L.; Hernandez-Munoz, R. Is Liver Enzyme Release Really Associated with Cell Necrosis Induced by Oxidant Stress? Oxid. Med. Cell Longev. 2016, 2016, 3529149. [CrossRef] [PubMed]

6. Sharaf El Din, U.A.A.; Salem, M.M.; Abdulazim, D.O. Uric acid in the pathogenesis of metabolic, renal, and cardiovascular diseases: A review. J. Adv. Res. 2017, 8, 537-548. [CrossRef]

7. Lanaspa, M.A.; Sanchez-Lozada, L.G.; Choi, Y.J.; Cicerchi, C.; Kanbay, M.; Roncal-Jimenez, C.A.; Ishimoto, T.; Li, N.; Marek, G.; Duranay, M.; et al. Uric acid induces hepatic steatosis by generation of mitochondrial oxidative stress: Potential role in fructose-dependent and -independent fatty liver. J. Biol. Chem. 2012, 287, 40732-40744. [CrossRef]

8. National Health and Nutrition Examination Survey (NHANES), National Health and Nutrition Examination Survey (NHANES), National Center for Health Statistics. 2017. Available online: https://wwwn.cdc.gov/nchs/nhanes/analyticguidelines.aspx (accessed on 10 May 2021).

9. The Practical Guide Identification, Evaluation, and Treatment of Overweight and Obesity in Adults. NHLBI Obesity Education Initiative, National Institute of Health. Available online: https:/ / www.nhlbi.nih.gov/files/docs/guidelines/prctgdc.pdf (accessed on 10 May 2021).

10. Adult Treatment Panel III (ATP III) Guidelines At-a-Glance Quick Desk Reference. National Cholesterol Education Program (NCEP). Available online: https:/ / www.nhlbi.nih.gov/files/docs/guidelines/atglance.pdf (accessed on 10 May 2021).

11. Stata Statistical Software: Release 13; Software; StataCorp, LP.: College Station, TX, USA, 2013.

12. Aragon, G.; Younossi, Z.M. When and how to evaluate mildly elevated liver enzymes in apparently healthy patients. Cleve. Clin. J. Med. 2010, 77, 195-204. [CrossRef]

13. Deb, S.; Puthanveetil, P.; Sakharkar, P. A Population-Based Cross-Sectional Study of the Association between Liver Enzymes and Lipid Levels. Int. J. Hepatol. 2018, 2018, 1286170. [CrossRef]

14. Sakharkar, P.; Deb, S. Examining Liver Function in Adults with Diabetes in the United States. J. Pharm. Pharm. Sci. 2021, 24, 317-328. [CrossRef] [PubMed]

15. Kong, L.; Liang, J.; Yang, L.; An, S.; Gao, C.; Liao, X.; Zhao, Y.; Yang, B. Reversing cytotoxicity of uric acid by supramolecular encapsulation with acyclic cucurbit[n] uril. Biomed. Mater. 2020, 16, 035025. [CrossRef] [PubMed]

16. Afzali, A.; Weiss, N.S.; Boyko, E.J.; Ioannou, G.N. Association between serum uric acid level and chronic liver disease in the United States. Hepatology 2010, 52, 578-589. [CrossRef] [PubMed]

17. Sirota, J.C.; McFann, K.; Targher, G.; Johnson, R.J.; Chonchol, M.; Jalal, D.I. Elevated serum uric acid levels are associated with non-alcoholic fatty liver disease independently of metabolic syndrome features in the United States: Liver ultrasound data from the National Health and Nutrition Examination Survey. Metabolism 2013, 62, 392-399. [CrossRef] [PubMed]

18. Zhou, M.; Yang, N.; Xing, X.; Chang, D.; Li, J.; Deng, J.; Chen, Y.; Hu, C.; Zhang, R.; Lu, X.; et al. Obesity interacts with hyperuricemia on the severity of non-alcoholic fatty liver disease. BMC Gastroenterol. 2021, 21, 43. [CrossRef]

19. Shih, M.H.; Lazo, M.; Liu, S.H.; Bonekamp, S.; Hernaez, R.; Clark, J.M. Association between serum uric acid and nonalcoholic fatty liver disease in the US population. J. Formos. Med. Assoc. 2015, 114, 314-320. [CrossRef] 
20. Vos, M.B.; Colvin, R.; Belt, P.; Molleston, J.P.; Murray, K.F.; Rosenthal, P.; Schwimmer, J.B.; Tonascia, J.; Unalp, A.; Lavine, J.E.; et al. Correlation of vitamin E, uric acid, and diet composition with histologic features of pediatric NAFLD. J. Pediatr. Gastroenterol. Nutr. 2012, 54, 90-96. [CrossRef]

21. Catanzaro, R.; Sciuto, M.; He, F.; Singh, B.; Marotta, F. Non-alcoholic fatty liver disease: Correlation with hyperuricemia in a European Mediterranean population. Acta Clin. Belg. 2020. [CrossRef]

22. Bao, T.; Ying, Z.; Gong, L.; Du, J.; Ji, G.; Li, Z.; Gao, W.; Jiang, X.; Yang, H.; Huang, Y.; et al. Association between Serum Uric Acid and Nonalcoholic Fatty Liver Disease in Nonobese Postmenopausal Women: A Cross-sectional Study. Sci Rep. 2020, 10, 10072. [CrossRef]

23. Xu, C.; Yu, C.; Xu, L.; Miao, M.; Li, Y. High serum uric acid increases the risk for nonalcoholic Fatty liver disease: A prospective observational study. PLoS ONE 2010, 5, e11578. [CrossRef]

24. Jensen, T.; Niwa, K.; Hisatome, I.; Kanbay, M.; Andres-Hernando, A.; Roncal-Jimenez, C.A.; Sato, Y.; Garcia, G.; Ohno, M.; Lanaspa, M.A.; et al. Increased Serum Uric Acid over five years is a Risk Factor for Developing Fatty Liver. Sci. Rep. 2018, 8 , 11735. [CrossRef]

25. Evangelopoulos, A.A.; Vallianou, N.G.; Panagiotakos, D.B.; Georgiou, A.T.; Zacharias, G.A.; Vogiatzakis, E.D.; Avgerinos, P.C. The Association Between Uric Acid and Hepatic Function Markers With the Metabolic Syndrome in Middle-aged, Overweight, and Obese People. Endocrinologist 2010, 20, 312-315. [CrossRef]

26. Lee, Y.J.; Lee, H.R.; Lee, J.H.; Shin, Y.H.; Shim, J.Y. Association between serum uric acid and non-alcoholic fatty liver disease in Korean adults. Clin. Chem. Lab. Med. 2010, 48, 175-180. [CrossRef] [PubMed]

27. Oral, A.; Sahin, T.; Turker, F.; Kocak, E. Relationship Between Serum Uric Acid Levels and Nonalcoholic Fatty Liver Disease in Non-Obese Patients. Medicina 2019, 55, 600. [CrossRef] [PubMed]

28. Zelber-Sagi, S.; Ben-Assuli, O.; Rabinowich, L.; Goldstein, A.; Magid, A.; Shalev, V.; Shibolet, O.; Chodick, G. The association between the serum levels of uric acid and alanine aminotransferase in a population-based cohort. Liver Int. 2015, 35, $2408-2415$. [CrossRef]

29. Brennan, P.; Clare, K.; George, J.; Dillon, J.F. Determining the role for uric acid in non-alcoholic steatohepatitis development and the utility of urate metabolites in diagnosis: An opinion review. World J. Gastroenterol. 2020, 26, 1683-1690. [CrossRef]

30. Hafner, M.; Rezen, T.; Rozman, D. Regulation of hepatic cytochromes p450 by lipids and cholesterol. Curr. Drug Metab. 2011, 12, 173-185. [CrossRef]

31. Ruggiero, C.; Cherubini, A.; Ble, A.; Bos, A.J.; Maggio, M.; Dixit, V.D.; Lauretani, F.; Bandinelli, S.; Senin, U.; Ferrucci, L. Uric acid and inflammatory markers. Eur. Heart J. 2006, 27, 1174-1181. [CrossRef]

32. Ballestri, S.; Nascimbeni, F.; Romagnoli, D.; Lonardo, A. The independent predictors of non-alcoholic steatohepatitis and its individual histological features.: Insulin resistance, serum uric acid, metabolic syndrome, alanine aminotransferase and serum total cholesterol are a clue to pathogenesis and candidate targets for treatment. Hepatol. Res. 2016, 46, 1074-1087. [CrossRef] [PubMed]

33. Lonardo, A.; Ballestri, S.; Marchesini, G.; Angulo, P.; Loria, P. Nonalcoholic fatty liver disease: A precursor of the metabolic syndrome. Dig. Liver Dis. 2015, 47, 181-190. [CrossRef] [PubMed]

34. Mantovani, A. NAFLD and risk of cardiac arrhythmias: Is hyperuricemia a neglected pathogenic mechanism? Dig. Liver Dis. 2018, 50, 518-520. [CrossRef]

35. Tani, S.; Matsuo, R.; Imatake, K.; Suzuki, Y.; Takahashi, A.; Yagi, T.; Matsumoto, N.; Okumura, Y. The serum uric acid level in females may be a better indicator of metabolic syndrome and its components than in males in a Japanese population. J. Cardiol. 2020, 76, 100-108. [CrossRef] [PubMed] 